\title{
Dynamic measurements of plastic deformation in a water-filled aluminum tube in response to detonation of a small explosives charge
}

\author{
Harold Sandusky ${ }^{a}$, Paul Chambers ${ }^{\text {a,* }}$, \\ Frank Zerilli ${ }^{a}$, Larry Fabini ${ }^{b}$ and \\ William Gottwald ${ }^{\mathrm{c}}$ \\ ${ }^{a}$ Naval Surface Warfare Center/Indian Head Division, \\ Code 920, 10901 New Hampshire Avenue, \\ Silver Spring, MD 20903, USA \\ ${ }^{\mathrm{b}}$ Naval Research Laboratory, Silver Spring, \\ MD 20903, USA \\ ${ }^{\mathrm{c}}$ Naval Surface Warfare Center/Carderock Division, \\ Silver Spring, MD 20903, USA
}

Received 19 January 1997

Revised 9 April 1999

Experiments have been conducted to benchmark computer code calculations for the dynamic interaction of explosions in water with structures. Aluminum cylinders with a length slightly more than twice their diameter were oriented vertically, sealed on the bottom by a thin plastic sheet, and filled with distilled water. An explosive charge suspended in the center of the tube plastically deformed but did not rupture the wall. Tube wall velocity, displacement, and strain were directly measured. The agreement among the three sets of dynamic data and the agreement of the terminal displacement measurements with the residual deformation were excellent.

Keywords: DYSMAS, shock validation, wall deformation, underwater detonation, computer modeling, streak photography, laser interferometry

\section{Introduction}

Computer modeling codes can provide solutions to large or complex events that are not easily tested. Since

\footnotetext{
${ }^{*}$ Corresponding author.
}

approximations to physical and chemical processes are required in the codes, they must be validated by precise experiments prior to the intended application. Of interest to this study is the complex interaction between a shock wave in water and a deformable structure, given that the properties of the explosive and the water are known. While there is some challenge in accurately modeling the high strain rate deformation of the structure from a simple shock impulse, the problem is considerably more complex because differences in shock impedances between water and structure permit a momentary separation (cavitation) during the interaction.

The Guirguis Hydro-Bulged Cylinder experiment was conceived for model validation by Guirguis [6]. The radially-symmetric arrangement consists of a water-filled tube with a centrally-positioned explosive charge. The symmetrical interior loading removes the complication of bubble collapse driving a water jet towards the structure, which occurs in the usual exterior loading. Interior loading also permits easy access to the deforming structure by various optical techniques for dynamic measurements.

The experimental data have been modeled in a preliminary way with the EPIC code of Johnson and Cook [8], which is Lagrangian. Computations are made on cells fixed to the material versus an Eulerian scheme where computations are made on spatially fixed cells through which mass passes. Both schemes have advantages that have been incorporated into the coupled Eulerian-Lagrangian DYSMAS code [4], which is the code to be validated.

\section{Experimental arrangement and data analysis}

The experimental arrangement is illustrated in Fig. 1. The deformable structure was a $9^{\prime \prime}$ long $\times 4^{\prime \prime}$ outer di- 


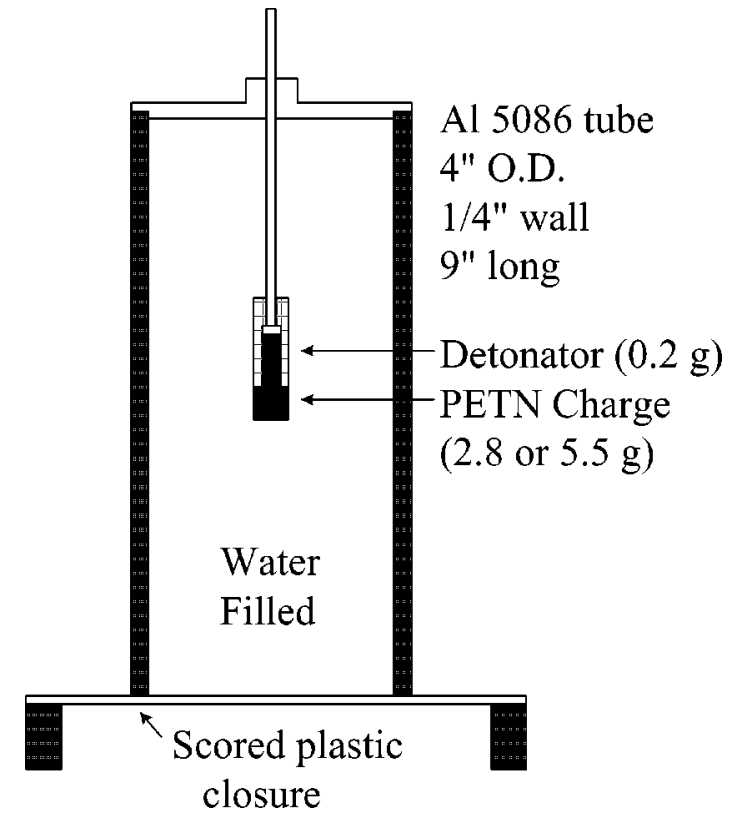

Fig. 1. Experimental arrangement.

ameter Al 5086 tube with a $0.25^{\prime \prime}$ wall thickness. The runout (roundness) and thickness of the tube wall varied by only $0.002^{\prime \prime}$. The tube was sealed at the bottom by a $1 / 10^{\prime \prime}$ thick polymethylmethacrylate (PMMA) sheet with a scored circle corresponding to the inner diameter of the tube. With this weakened bottom closure and the tube being mostly open on top, there was little resistance to the de-oxygenated water that initially filled the tube from being ejected by the exploding charge. A pentaerythritol tetranitrate (PETN) explosive pellet was suspended in the center of the tube. The 2.8 and $5.5 \mathrm{~g}$ explosive charges were selected to achieve final wall strains of 10 and $20 \%$, respectively, based on preliminary EPIC (Johnson and Cook [8]) and DYSMAS [4] calculations and rapid response bound estimates by Zilliacus [12]. At these strains the tube would deform plastically without rupturing. A 200-mg PETN detonator was positioned above the charge and encased in PMMA, with the leads extending up through a hypodermic needle tube. The needle tubing was further filled with epoxy to prevent collapse during shock loading, thereby simplifying the modeling.

The experiment was conducted in a firing chamber with the arrangement and instrumentation positioned as shown in Fig. 2. The dynamic measurements of tube deformation were at the same cross-sectional plane (tube mid-height) as the charge, but at different positions to avoid interference with each other. Relating the various measurements required that the charge be ac- curately centered and that the tube have uniform mechanical properties, which was the case based on the consistent residual deformations that are reported in the next section.

Dynamic wall displacement was measured directly by a Cordin Model 136A streak camera. The streak camera used $60 \mathrm{~mm}$ wide imaging optics, set to $4 \times$ magnification. The slit view of the camera at the tube was $15 \mathrm{~mm}$ wide. The writing speed was set to either 0.2 or $0.4 \mathrm{~mm} / \mu$ s to obtain an optimum $45^{\circ}$ trace for the expected event, with a temporal accuracy of $\sim 0.1 \mu \mathrm{s}$. The tube was backlit to produce an image of the wall displacement as a function of time. The image was then digitized to yield the wall deformation profile directly. Velocity versus time was obtained by fitting a small group of the digitized data points to a quadratic equation, differentiating the equation to obtain velocity at the center of the group, shifting the group by one data point, and repeating the process.

Wall velocity was measured simultaneously by a fiber optic velocity sensor (Fabiny and Kersey [5]) viewing the opposite side of the tube $\left(180^{\circ}\right)$ as the streak camera. The sensor operated through detection of the Doppler-induced frequency shift of light reflected from the surface. A fiber optic interferometer is used to transpose the frequency shift into an interferometric phase shift. Light from a single frequency $\mathrm{Nd}$ :YAG laser, operating at $1.319 \mu \mathrm{m}$, is passed along a fiber to a collimating lens for directing the laser light to the moving target. Collimation is by a simple GRIN rod lens, with a low inherent back reflection of $<-40 \mathrm{~dB}$. A small strip of retroreflecting tape was used on the wall surface to ensure that a sufficient signal was returned as the moving surface undergoes angular deviations. Light reflected from the target was directed via the input fiber and a coupler to an unbalanced Mach-Zehnder interferometer. The phase change of the interferometer output is determined by:

$$
\Phi=(4 \pi n d V) /(c \lambda)
$$

where $n$ is the refractive index of the fiber, $d$ is the Mach-Zehnder path difference, $V$ is the surface velocity, $c$ is the speed of light, and $\lambda$ is the laser wavelength. The responsivity, e.g., $\Phi / V$, is given by the cofactor $(4 \pi n d / c \lambda)$ which is directly related to the path imbalance of the interferometer. The technique used to decode the optical phase shift induced in the MachZehnder interferometer was based on the phase generated carrier method in which a carrier signal, in this case $1 \mathrm{MHz}$, modulates the phase in one arm of the in- 


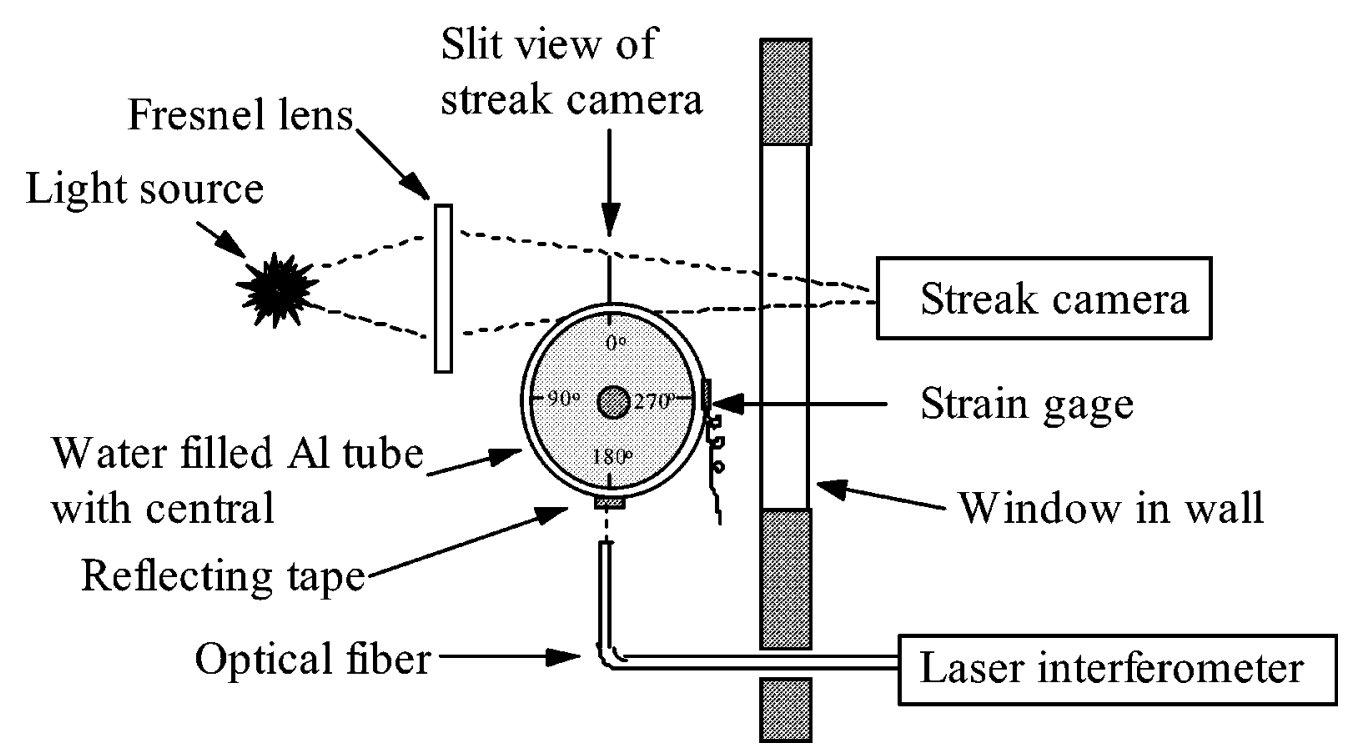

Fig. 2. Top view of instrumented apparatus at the tube's mid-height plane.

terferometer. Wall displacement as a function of time was was obtained by integrating the velocity data.

Three types of strain gages were applied. One was a constantan gage bonded with cyanoacrylate (3-5\% $\left.\varepsilon_{\max }\right)$ and connected to a Wheatstone bridge. The second was an annealed constantan gage which was bonded with epoxy $\left(5-15 \% \varepsilon_{\max }\right)$ and connected to a constant current power supply. The third was an optical strain gage from the Naval Research Laboratory (NRL) consisting of a fiber with an internal grating.

\section{Experimental results and discussion}

The response of the Al tube to a total $3.0 \mathrm{~g}$ charge is shown in Fig. 3. The traces for wall velocity and displacement versus time shown in Fig. 3(a) and (b), respectively, show excellent agreement between the two independent measuring techniques. The Al cylinder wall reached its peak velocity in $\sim 10 \mu$ s after the wall first encountered the shock wave from the water. Then, internal forces in the Al began to arrest the wall motion. During the shock loading of the wall, it separated from the water producing a cavity between them. As the wall velocity declined, the water was able to catch up and reinteract with the wall, producing the velocity spike at $\sim 80 \mu \mathrm{s}$. After $110 \mu \mathrm{s}$, the cylinder wall reached its maximum deformation, at which point internal restraining forces caused the wall to contract again, then expand, and contract producing an oscillating or ringing effect. While not shown, wall displacement measurements were also replicated by a circumferentially-oriented strain gage for the first $7 \mu \mathrm{s}$, when either the cyanoacrylate bond or the gage itself failed.

Plots of residual wall deformation along two sides of the tube are shown in Fig. 3(c). The agreement of the deformation on the side viewed by the streak camera $\left(0^{\circ}\right)$ with the deformation on the side viewed by the interferometer $\left(180^{\circ}\right)$ justifies comparing the two dynamic measurements from opposite sides of the tube. This agreement along with the symmetry about the tube mid-height also verifies the centering of the charge in the cylinder. As shown in Fig. 4, reproducible deformation was obtained in a replicated experiment in which the recording of dynamic measurements had failed.

The responses of the same size Al tube to a total 5.7 g charge (Shot SV-4) is compared in Fig. 5 to the previously shown response for a $3.0 \mathrm{~g}$ charge in Fig. 3 . The increased shock from the larger charge caused the reflecting tape for the interfermometer to separate from the wall, thus only streak camera data are shown for the dynamic measurements in Figs 5(a) and (b). As can be seen from Fig. 5(a), the peak wall velocity for Shot SV-4 is about 1.5 times higher than the peak wall velocity for Shot SV-3. This effect was expected since the momentum is proportional to the square root of the energy release. Further, the basic features of both velocity curves are similar, including the times at which the two peaks were reached. As is evident from Fig. 5(b), the final wall deformation of the cylinder for Shot SV-4 is 

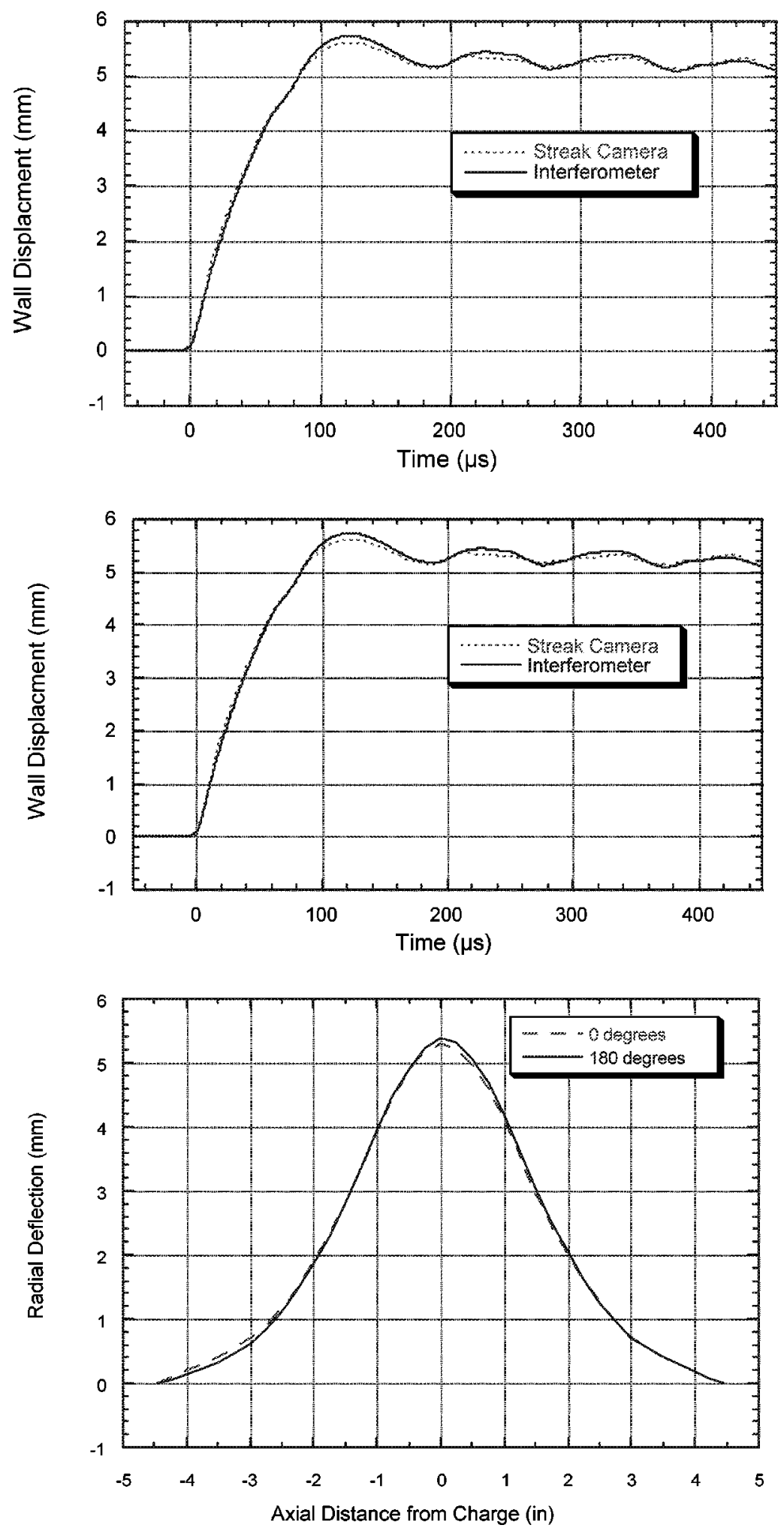

Fig. 3. Outside wall measurements from detonating a 3.0 g charge (Shot SV-3). (a)-(b) Dynamic responses at same tube height as center of charge; (c) Permanent deformation. 


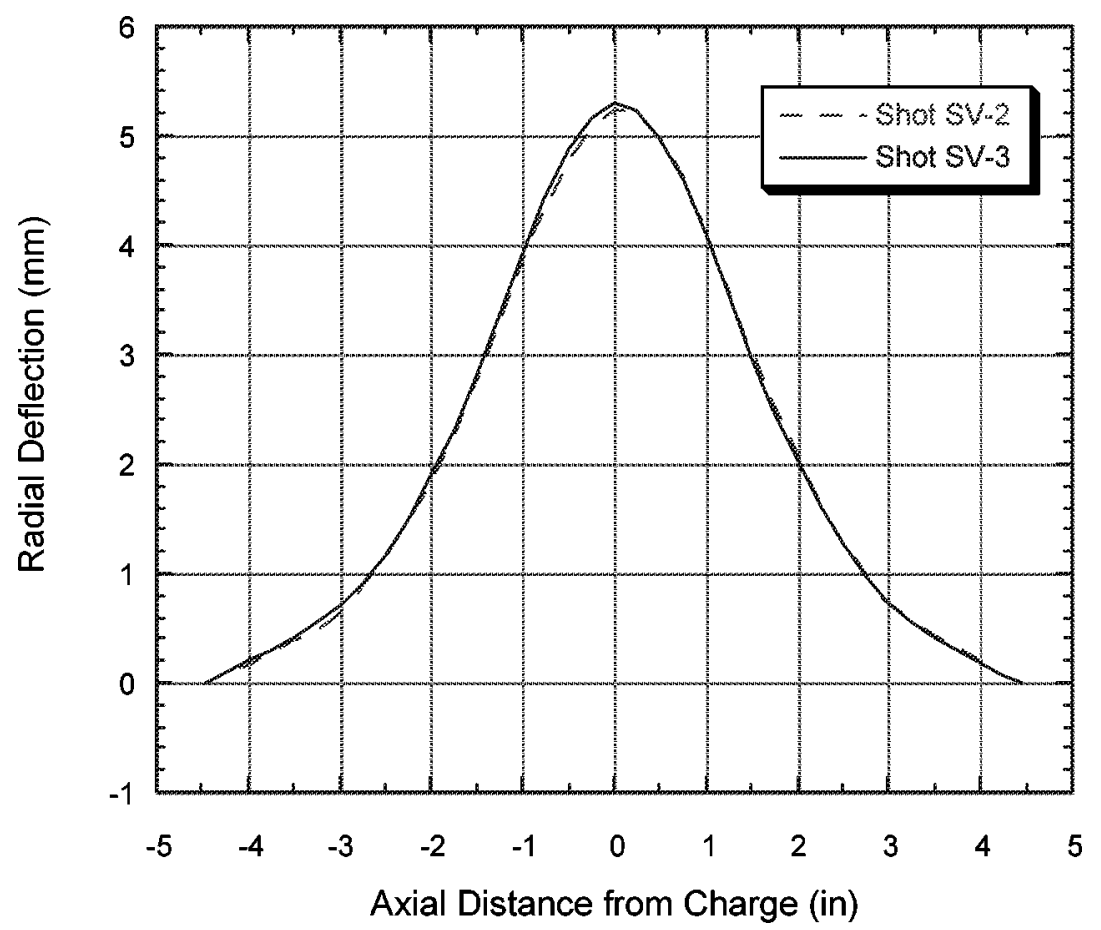

Fig. 4. Comparison of final wall deformation along the $0^{\circ}$ side for replicated experiments, Shots SV-2 and SV-3.

approximately twice the final deformation of the cylinder in Shot SV-3, in proportion to the charge masses. Maximum deformation for the $5.7 \mathrm{~g}$ PETN charge occurred later $(\sim 170 \mu \mathrm{s})$ than for the $3 \mathrm{~g}$ PETN charge $(129 \mu \mathrm{s})$. These two sets of data are therefore consistent and reasonable given the ratio of explosive charge sizes used in the two experiments.

Other strain gage techniques in Shot SV-4 were not anymore successful than in Shot SV-3 for determining wall displacement. The NRL optical gage replicated wall displacement measurements for only $8 \mu$ s before failing. Epoxy-bonded, annealed constantan gages, oriented both axially and circumferentially survived for $\sim 40 \mu$ s but the data obtained from them were poorly structured.

Plots of residual wall deformation along one side of each tube in Fig. 5(c) also show about twice the displacement for the larger charge along the length of the tube. With the larger charge in Shot SV-4, deformation occurred more noticeably all the way to the ends of the tube. Displacements vanish at the ends of the tube because the water is essentially unconfined, allowing pressures to be instantaneously relieved. Table 1 shows the wall thicknesses, measured at the tube midheight, and tube heights for both cylinders measured before and after the shots. The wall for the tube used in Shot SV-4 was thinned more than that in Shot SV-3.
Table 1

Wall thicknesses at the midplane and tube heights measured before and after the shots

\begin{tabular}{|c|c|c|c|c|}
\hline \multirow[t]{2}{*}{ Shot } & \multicolumn{2}{|c|}{ Wall thickness (in) } & \multicolumn{2}{|c|}{ Tube height (in) } \\
\hline & Before & After & Before & After \\
\hline SV-3 & 0.255 & 0.234 & 8.999 & 8.934 \\
\hline SV-4 & 0.255 & 0.222 & 9.009 & 8.870 \\
\hline
\end{tabular}

Moreover, the tube was shorter after Shot SV-4, but retained its height in Shot SV-3. If the wall deformations were purely circumferential strain, the wall would have thinned even more than observed for Shot SV-4. The tube is bending to some extent, which agrees with the shortening of the tube for the larger charge shot.

\section{Numerical simulations}

Numerical simulations of the cylinder response for purposes of designing the experiment were performed with several material dynamics computer programs. EPIC (Johnson and Cook [8]) simulations initially used material strength data for Al 5083 provided by Chen and Gray [1], fitted to the Zerilli and Armstrong [11] constitutive equation for FCC metals:

$$
\sigma=c_{0}+B \sqrt{\varepsilon \mathrm{e}^{-\beta T}},
$$



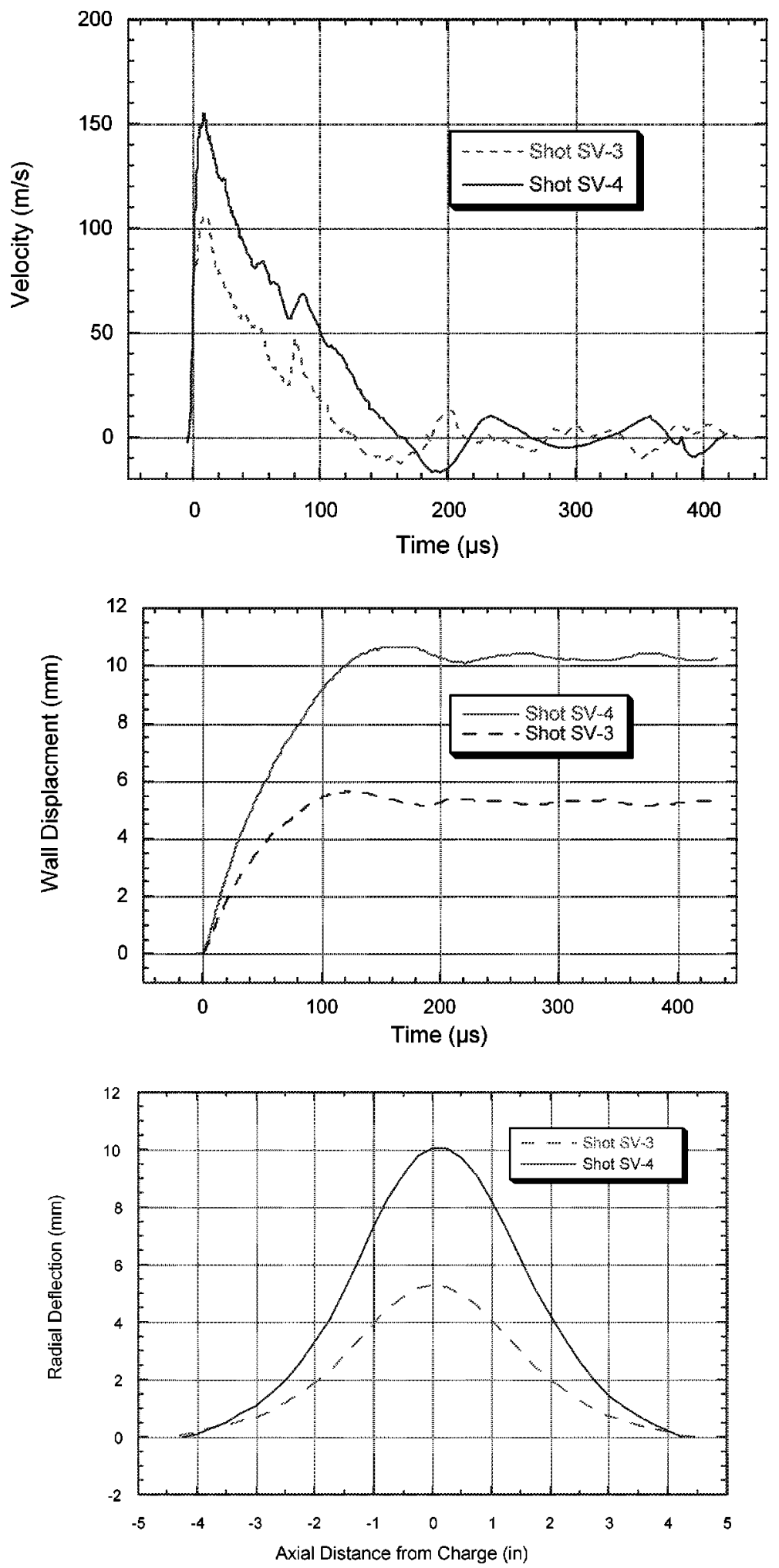

Fig. 5. Outside wall measurements from detonating a $5.7 \mathrm{~g}$ charge (Shot SV-4) versus a $3.0 \mathrm{~g}$ charge (Shot SV-3). (a)-(b) Dynamic responses at same tube height as center of charge; (c) Permanent deformation along $0^{\circ}$ side. 
Table 2

\begin{tabular}{lc}
$\begin{array}{l}\text { Zerilli-Armstrong parameters } \\
\text { used in numerical simulations }\end{array}$ \\
\hline$c_{0}(\mathrm{MPa})$ & 234 \\
$B(\mathrm{MPa})$ & 1283 \\
$\beta_{0}\left(\mathrm{~K}^{-1}\right)$ & 0.00371 \\
$\beta_{1}\left(\mathrm{~K}^{-1}\right)$ & 0.000153 \\
\hline
\end{tabular}

Table 3

JWL parameters for PETN

\begin{tabular}{lc}
\hline$\rho\left(\mathrm{kg} / \mathrm{m}^{3}\right)$ & 1770 \\
$D(\mathrm{~m} / \mathrm{s})$ & 8300 \\
$A(\mathrm{GPa})$ & 613.27 \\
$B(\mathrm{GPa})$ & 15.069 \\
$R_{1}$ & 4.4 \\
$R_{2}$ & 1.2 \\
$\omega$ & 0.25 \\
$E_{0}\left(\mathrm{GJ} / \mathrm{m}^{3}\right)$ & 10.1 \\
\hline
\end{tabular}

where $\beta=\beta_{0}-\beta_{1} \ln \varepsilon$. The fitted parameters for $\mathrm{Al}$ 5083 are given in Table 2 . The JWL equation:

$$
\begin{aligned}
P= & A\left(1-\frac{\omega}{R_{1} V}\right) \mathrm{e}^{-R_{1} V} \\
& +B\left(1-\frac{\omega}{R_{2} V}\right) \mathrm{e}^{-R_{2} V}+\omega \frac{E_{0}}{V},
\end{aligned}
$$

where $V=\rho_{0} / \rho$ was used to describe PETN. The JWL parameters for PETN (Guirguis [7]) are given in Table 3. When compared with Shot SV-3, the predicted deformation was approximately $25 \%$ smaller than that shown in Fig. 3.

The Al 5086 (4.0 Mg-0.4 Mn-0.15 Cr) used in the experiment is very similar to $\mathrm{Al} 5083$ (4.4 Mg-0.7Mn$0.15 \mathrm{Cr}$ ), with Al 5086 having a slightly smaller solute content. Thus the principal difference between the Al 5083 which was previously characterized and the Al 5086 used in the test would be in the temper of the material with a small difference due to the solute content. In the Zerilli-Armstrong equation, the effect of solute content and temper (which affects the grain size) is principally contained in the constant $c_{0}$. Thus any difference in solute content and temper should be able to be accounted for by a change in the value of $c_{0}$ (which, for FCC metals, is the yield stress). Recently, strength measurements were made on samples of the same tube from which the tested cylinders were cut (Chen and Gray [2]). Analysis of this data indicates that a value of $c_{0}$ of $208 \mathrm{MPa}$, together with the remaining parameters in Table 2 gives a good representation of the plastic flow properties of Al 5086.
As shown in Fig. 6, this smaller yield stress does not completely account for the underprediction of the deformation. A reasonably good fit can be obtained by increasing the heat of detonation for PETN from the published experimental value (Dobratz [3]) of $10.1 \mathrm{GJ} / \mathrm{m}^{3}$ to the value $12.1 \mathrm{GJ} / \mathrm{m}^{3}$ calculated from the $\mathrm{H}_{2} \mathrm{O}-\mathrm{CO}_{2}$ arbitrary. This approach, however, is not recommended for code validation with a well-controlled experiment. Future work will utilize the DYSMAS code, which is designed to handle the complex water/wall interactions.

\section{Summary and conclusions}

Shock validation experiments were conducted on 9-inch long Al 5086 water filled tubes using PETN charges in order to authenticate results obtained from the hydrocode DYSMAS. Dynamic cylinder wall deformation was measured directly with a streak camera, while wall velocity was measured directly with a fiber optic interferometrically decoded Doppler velocimeter. Comparison of wall velocities and radial displacements as measured by the two techniques for the same experiment gave excellent agreement. Moreover, the agreement between residual wall deformation for replicated experiments also agreed well, demonstrating the repeatability of our technique. Additionally, radial displacements were found to be symmetrical about the cylinder axis and in good agreement on opposite sides of the deformed cylinders.

A more powerful $5.7 \mathrm{~g}$ PETN charge was shown to produce a higher cylinder wall velocity than the smaller 3 g PETN charge while qualitatively reproducing the same features of displacement velocity as a function of time. The radial displacement was seen to be roughly double of that produced by the smaller charge. Although strain gages attached to the cylinders did not survive long, their data agreed well with the streak camera and optical fiber velocimetry results.

Overall, the consistency, symmetry, and reproducibility of these experiments indicate that they should provide excellent results with which to validate the underwater explosion modeling code DYSMAS. Future experiments will attempt to measure the pressure directly on the inner cylinder wall during and subsequent to shock interaction. Additionally, an attempt will be made to measure the pressure at the water/tube interface to record complex fluid/structure interaction in which fluid cavitation occurs. Experiments using dif- 


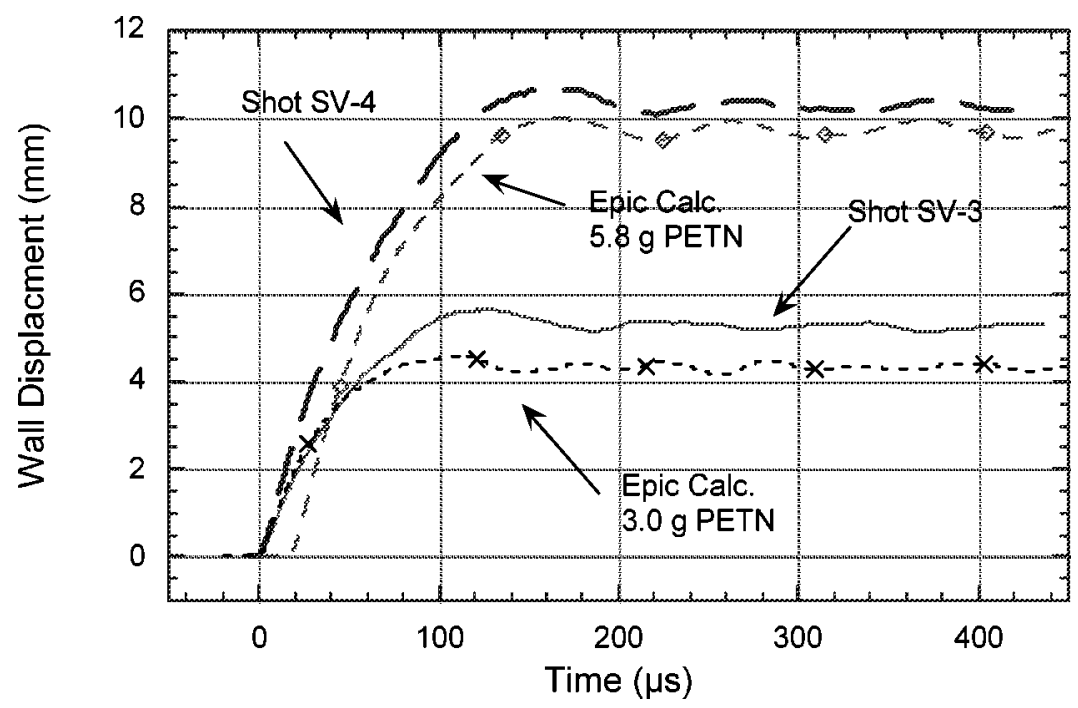

Fig. 6. Comparison of EPIC calculations with dynamic responses from Shots SV-3 and SV-4.

ferent size cylinders and different materials are also planned. These data, taken together with our present results, should be adequate to provide a complete validation of DYSMAS code results.

\section{Acknowledgements}

This work was conducted for the U.S./German project agreement on computer codes for underwater explosion effects and supported by the Office of Naval Research.

\section{References}

[1] S.R. Chen and G.T. Gray, III, Los Alamos National Laboratory, Private communication (November 1995).

[2] S.R. Chen and G.T. Gray, III, Los Alamos National Laboratory, Private communication (September 1996).
[3] LLNL Explosives Handbook, B.M. Dobratz, ed., Lawrence Livermore Laboratory, University of California, Livermore UCRL-52997, 1981

[4] Dynamic systems mechanics advanced simulation coupled Eulerian-Langrangian hydrocode, IAEG, Ottobrunn, Germany (April 1995).

[5] L. Fabiny and A.D. Kersey, in: Proc. of 1996 Optical Fiber Sensor Conf.

[6] R. Guirguis, NSWC/IHD, Private communication (July 1995).

[7] R. Guirguis, NSWC/IHD, Private communication (October 1996).

[8] G.R. Johnson and W.H. Cook, in: Proc. of the 7th Int. Symposium on Ballistics, The Hague, The Netherlands, 1983, p. 541.

[9] H.D. Jones and F.J. Zerilli, NSWC/IHD, Private communication (October 1996)

[10] H.D. Jones and F.J. Zerilli, J. Appl. Phys. 69 (1991), 389.

[11] F.J. Zerilli and R.W. Armstrong, J. Appl. Phys. 61 (1987), 1816.

[12] S. Zilliacus, NSWC/CD, Private communication (October 1996). 

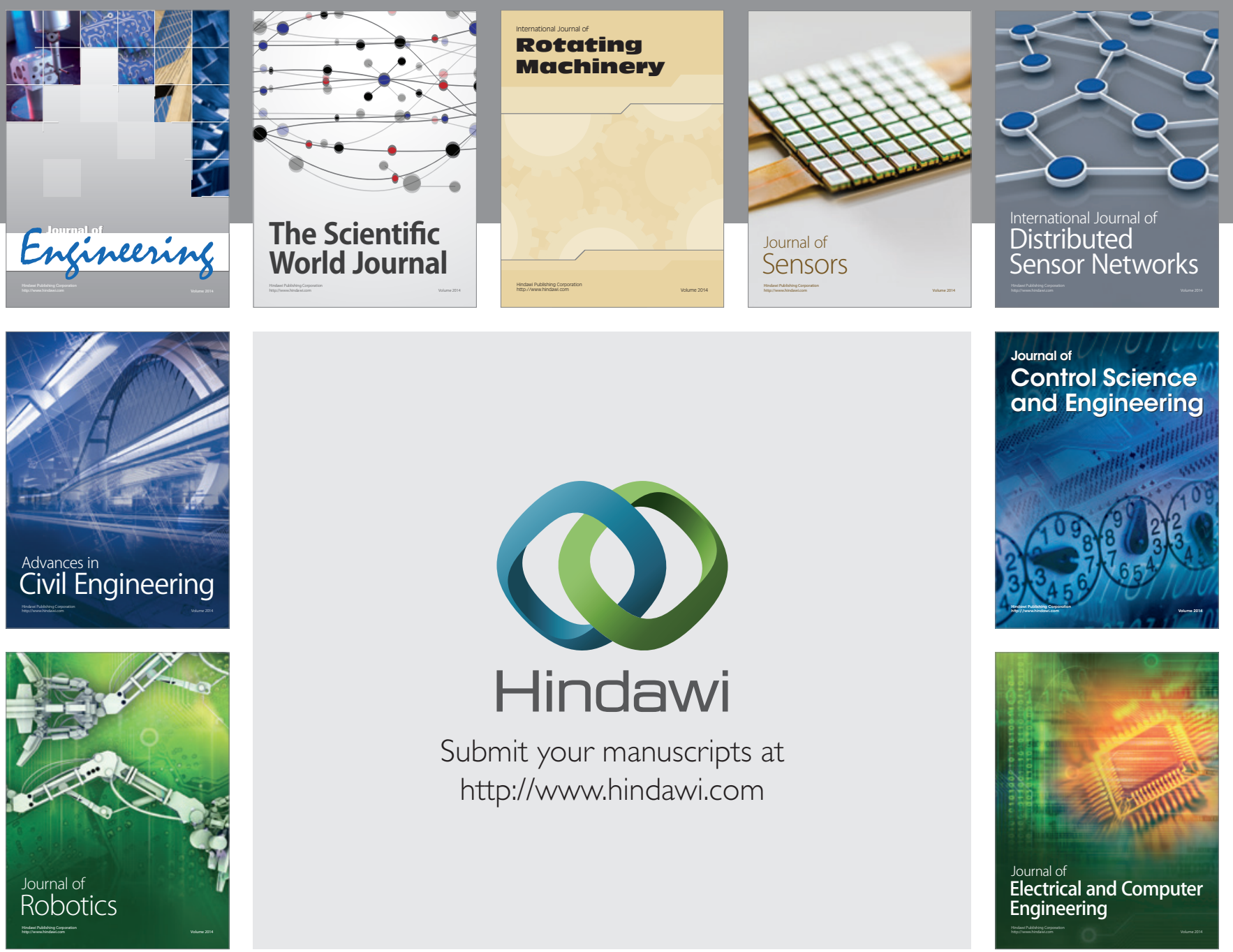

Submit your manuscripts at

http://www.hindawi.com
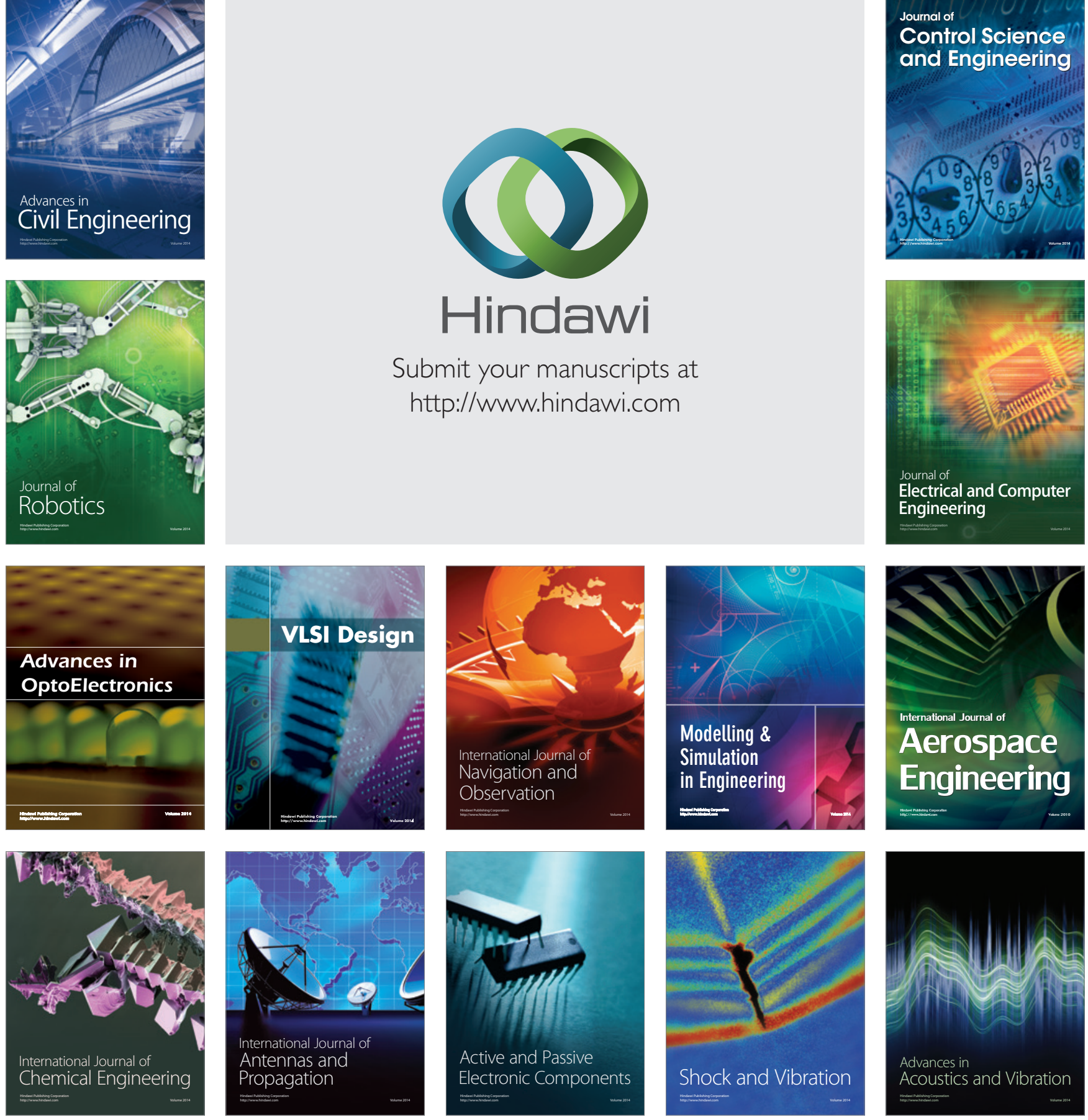